\title{
Grenz rays: an alternative treatment for superficial skin cancers in elderly patients
}

\section{Renato G Panizzon ${ }^{\dagger}$}

Grenz rays are electromagnetic waves (i.e., x-rays of very low energy and situated in the electromagnetic spectrum between UV and $x$-rays). This kind of $x$-ray is suitable for very superficial processes in the skin. This means that lesions up to a thickness of $1 \mathrm{~mm}$ can be irradiated. For these reasons, chronic dermatoses, precancerous lesions or superficial skin cancers can be treated. Since the introduction of this very superficial and low-energy treatment modality, Grenz rays have shown no serious side effects. Good indications for a Grenz ray treatment are psoriasis of the scalp and of the nails, chronic eczemas, disseminated superficial actinic porokeratosis, Bowen's disease, Paget's disease, lentigo maligna, superficial basal cell carcinoma or squamous cell carcinoma. The only side effects are slight hypo-/hyper-pigmentation of the skin and it has to be noticed that there is no hair loss after Grenz ray treatment, and no side effects to deeper situated structures.

Grenz rays are electromagnetic waves lying in the spectrum between UV and x-rays. The $\mathrm{x}$-rays were discovered by Wilhelm C Roentgen in 1885 , but in 1910, Schultz described the very soft $x$-rays and Bucky called them low-energy $\mathrm{x}$-rays in 1923. In his honor, these rays are sometimes called 'Bucky rays'. Since these rays are lie at the border zone between UV and x-rays, and as the German translation of border is 'Grenze', these rays were also called 'grenz rays'. Another denomination is ultrasoft $\mathrm{x}$-rays. These Grenz rays are produced by machines operating mainly between 8 and $20 \mathrm{kV}$. Some $\mathrm{x}$-ray machines deliver soft x-rays, including Grenz rays. Nowadays, machines operate with a spectrum going from 10 to $100 \mathrm{kV}$ or $150 \mathrm{kV}$. There are only few manufacturers producing only Grenzray machines. Grenz rays have a half-value depth, in other words a $50 \%$ isodose, in the range of 0.5 to $1.0 \mathrm{~mm}$. This means that dermatoses or tumorous lesions of up to $1 \mathrm{~mm}$ thickness, especially the epidermal and upper dermal layers, can be treated with these $\mathrm{x}$-rays [1-3].

\section{Benign skin diseases}

Among the benign skin diseases, psoriasis and chronic eczemas should be mentioned $[1,4]$, as well as disseminated superficial actinic porokeratosis [5]. For these benign dermatoses, several treatment options are available, but since they are often chronic and recalcitrant, a second- or third-line treatment option with radiation therapy is welcome [6]. Among psoriasis, we know that the psoriasis of the scalp and of the nails are especially difficult to treat and we want to stress that for these lesions, treatment series of 2 Gy six times is helpful. The same treatment schedule can be used for chronic eczemas of the hands, especially in patients who were metalworkers and have chronic professional eczema. The advantage of Grenz rays is that a second or even a third treatment series can be applied, which is not possible if we use higher $\mathrm{x}$-ray energies ( $>20 \mathrm{kV}$, orthovoltage treatment). Finally, the disseminated superficial actinic porokeratosis, often not responding to topical treatments, will respond to this treatment.

In summary, the main indications among benign skin diseases treated with Grenz rays are psoriasis and eczemas.

\section{Malignant skin diseases}

Regarding indications for malignant skin diseases, extensive actinic keratoses should be mentioned, especially over the scalp or over the forearms, which respond well to a treatment of 4 Gy six times. In theses cases, erythematous reactions, towards the end of treatment, have to be considered and the patients must be informed before the treatment starts. Using the same method, Bowen's disease of the genital area can be treated with good success. Similarly, extramammary Paget's disease responds to this treatment [7]. Here we have to look for underlying carcinomas that are seldomly found. The excellent cosmetic result for lentigo malignas among elderly patients should be stressed [8]. In this case, the treatment schedule is a little bit different: we use higher single doses, that is 10 Gy $10-12$ times (twice per week) or 20 Gy 5-6 times (once per week), depending on the diameter of the lesion, with the latter used for smaller lesions (up to $2.5 \mathrm{~cm}$ ). Here again, the skin shows a heavy reaction at the end of the treatment, but there is

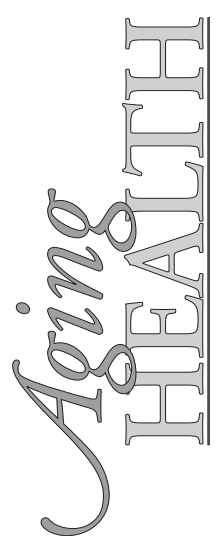

${ }^{+}$Department of Dermatology, University Hospital CHUV, CH-1011 Lausanne, Switzerland

Tel.: +41213143262

Fax: +41213140382

renato.panizzon@chuv.ch

\section{Keywords}

basal cell carcinoma $\bullet$ Bowen's disease $\bullet$ Bucky rays $\bullet$ eczema

- Grenz rays • lentigo maligna

- porokeratosis $\bullet$ precancerous

lesion • psoriasis • squamous cell

carcinoma $\bullet$ ultrasoft $x$-ray

- very superficial $x$-ray

\section{future ferot $_{\text {medicine }}$ fsg}


a good regression after several weeks. Since the half-value depth is $1 \mathrm{~mm}$ in atrophic older skin, the atypical melanocyte can be reached even if located in the hair follicles. Superficial basal cell carcinomas or superficial squamous cell carcinomas may also be treated with 6 Gy 12 times, twice per week [9]. On the other hand, one must be aware of possible deeper infiltration of skin cancers. In particular, squamous cell cancer may present a much deeper infiltration depending on their diameter and localization and the possible extension along hair follicles, nerves and so on. In these cases, orthovoltage radiotherapy can be the choice of energy in order to avoid underdosage of the lesion and an in-field recurrence. The advantage of Grenz rays is that we are able to repeat the treatment series if necessary since we observe only few long-term side effects, such as hyper- or hypo-pigmentation. There is no alopecia after treatment, and tumor development in the radiation field is only rarely observed [3]. This only happens if the lifetime total dose is over $100 \mathrm{~Gy}$, if fractionated doses are not used, if the minimum interval between series is not at least 6 months and if doses are not adapted to the surface area [3].

\section{Conclusion}

Disseminated actinic keratoses, Bowen's disease and lentigo maligna are ideal indications for Grenz ray treatment.

\section{Bibliography}

Papers of special note have been highlighted as:

- of interest

-• of considerable interest

1. Warner IA, Cruz PD: Grenz ray therapy in the new millennium: still a valid treatment option? Dermatitis 19, 73-80 (2008).

2. Olivo P: Grenz ray therapy of benign skin diseases. In: Radiation Treatment and Radiation Reactions in Dermatology. Panizzon RG, Cooper JS (Eds). Springer, NY, USA, 41-47 (2004).

-• Excellent overview regarding the use of Grenz rays in dermatology.

3. Lindelöf B, Eklund G: Incidence of malignant skin tumors in 14140 patients after Grenz ray treatment for benign skin disorders. Arch. Dermatol. 122, 1391-1395 (1986).

- Best review concerning the low incidence of tumors developing after the use of Grenz rays.

6. Sumila M, Notter M, Bodis S, Itin P, Strablenther. Onkol. 4, 218-223 (2008). lentigo maligna and lentigo maligna
In conclusion, Grenz ray therapy is a simple, affordable, painless and effective option to manage several refractory benign dermatoses and superficial tumors, and is a safe treatment modality if administrated according to the guidelines recommended. In combination with orthovoltage radiotherapy, Grenz ray therapy is the ideal choice in dermatological radiotherapy.

\section{Future perspective}

Since the general population is getting older and older, we expect an increase of chronic benign dermatoses as well as skin tumors. Therefore, we imagine that elderly patients will easily accept an $\mathrm{x}$-ray treatment. Grenz ray therapy is an excellent modality, especially if diagnosed early. The only condition is that the manufacturers include Grenz ray quality in their superficial or orthovoltage machines. In addition, more clinical trials with this therapy are needed.

\section{Financial \& competing interests disclosure}

The author has no relevant affiliations or financial involvement with any organization or entity with a financial interest in or financial conflict with the subject matter or materials discussed in the manuscript. This includes employment, consultancies, honoraria, stock ownership or options, expert testimony, grants or patents received or pending, or royalties.

No writing assistance was utilized in the production of this manuscript.

-• One of the first and largest study to demonstrate the excellent results of radiotherapy of lentigo maligna and lentigo maligna melanoma.

9. Bodner WR, Hilaris BS, Alagheband M et al: : Use of low energy $\mathrm{x}$-rays in the treatment of superficial non-melanoma skin cancer. Cancer Invest. 21, 355-362 (2003). Gruber G: Long term results of radiotherapy in patients with chronic eczema or psoriasis.

7. Moreno-Arias GA, Conill C, Sola-Casas MA et al.: Radiotherapy for in situ extramammary Paget disease of the vulva. J. Dermatol. Treat. 14, 119-123 (2003).

8. Farshad A, Burg G, Panizzon R, Dummer R: A retrospective study of 150 patients with melanoma and the efficacyof radiotherapy using Grenz or soft x-rays. Br. J. Dermatol. 146, 1042-1046 (2002).

\section{Affiliation}

- Renato G Panizzon, MD Department of Dermatology, University Hospital CHUV, CH-1011 Lausanne, Switzerland Tel.: +41213143262 Fax: +41 213140382 renato.panizzon@chuv.ch 